\title{
Iron-rich solar particle events measured by SOHO/ERNE
}

\section{O. Raukunen, E. Valtonen* and R. Vainio}

Department of Physics and Astronomy, University of Turku, 20014 Finland E-mail: bajraudutu.fi

\begin{abstract}
We have surveyed the SOHO/ERNE heavy ion data from the beginning of solar cycle 23 until the end of May 2015, well into solar cycle 24. We have used the long observation period to study the properties of heavy ions (from $\mathrm{C}$ to $\mathrm{Fe}$ ) and to compare the two solar cycles in this respect. We have surveyed the data for solar energetic particle events with enhancements in the $\mathrm{Fe} / \mathrm{C}$ and $\mathrm{Fe} / \mathrm{O}$ intensity ratios in energy range 5-15 MeV per nucleon, and made solar event (type III burst, flare, CME) associations for the selected events when possible. We have studied the properties of heavy ions in these events and made a comparison of the average relative abundances of heavy ions between the two solar cycles. We found that there were considerably fewer Fe-enhancements during the current solar cycle than during the previous one, but taking into account the difference in the observed overall level of solar activity, the difference is small. We also found that the average abundances of $\mathrm{Ne}-\mathrm{Fe}$ in the selected events of cycle 23 were noticeably higher than in the events of cycle 24 .
\end{abstract}

The 34th International Cosmic Ray Conference,

30 July- 6 August, 2015

The Hague, The Netherlands

\footnotetext{
* Speaker.
} 


\section{Introduction}

The earliest observations of solar energetic particle (SEP) events were done with ionization chambers in 1946 by Forbush [四]. The events, nowadays known as ground level events (GLEs), were presumed to be caused by solar flares. In a comprehensive review of radio observations, Wild et al. [2] suggested that the fast-drift type III radio bursts were produced by flare-accelerated outward streaming electrons, whereas slow-drift type II bursts were produced by electrons accelerated by shock waves that could accelerate also protons. This idea of two different physical mechanisms of SEP acceleration, that contribute to the two classes of solar particle events, namely impulsive and gradual events, has been valid ever since (e.g. [B]]). In the two-class paradigm impulsive events are associated with resonant stochastic acceleration during magnetic reconnection in solar flares, whereas gradual events are caused by diffusive acceleration at shock waves driven by coronal mass ejections (CMEs). Impulsive events have typically durations of a few hours, small fluences and compact spatial scales, compared to the duration of several days, larger fluences and extensive spatial scales of gradual events.

A key feature in distinguishing the two classes of solar particle events has been the differences in their energetic particle composition. In general, impulsive events are electron rich, have ${ }^{3} \mathrm{He} /{ }^{4} \mathrm{He}$ ratios enhanced by factor up to $10^{4}$ and $\mathrm{Fe} / \mathrm{O}$ ratios enhanced up to factor of 10 compared to coronal values (e.g. [四, 可]). Gradual events, on the other hand, have energetic particle composition similar to that of corona or solar wind. In reality, the separation of the two classes is not clean; residual suprathermal ions from impulsive events may contribute to the seed material accelerated by CMEdriven shock waves, resulting in intermediate values for ${ }^{3} \mathrm{He} /{ }^{4} \mathrm{He}$ or heavy element abundances (e.g. [四, 口, 四]).

\section{Observations}

We analysed the SOHO/ERNE heavy ion data from the beginning of solar cycle 23 until the end of May 2015, producing intensity-time profiles for C, O and Fe. There have been some breaks in observations of the instrument, most notably from 25 June 1998 to 9 October 1998, from 21 December 1998 to 8 February 1999 and from 9 December 2012 to 8 February 2013. These, along with all the shorter breaks, have been taken into account in the further analysis.

Figure $\mathrm{Wa}$ ) shows monthly and Figure $\mathrm{Wb}$ ) annual average intensities of 5-15 MeV/n C, O and $\mathrm{Fe}$ as well as the intensity ratios $\mathrm{Fe} / \mathrm{C}$ and $\mathrm{Fe} / \mathrm{O}$. The monthly plot shows large variations in the $\mathrm{Fe} / \mathrm{C}$ and $\mathrm{Fe} / \mathrm{O}$ ratios even for successive months; for example, the value of $\mathrm{Fe} / \mathrm{C}$ increased over 70 -fold from $0.020 \pm 0.003$ in September 2004 to $1.533 \pm 0.323$ in October 2004. The high value of $\mathrm{Fe} / \mathrm{C}$ ratio in October 2004 is explained by the fact that there was very little SEP activity during the month, except the particle event(s) of 30.10.2004, which happened to be iron-rich. These SEP events were associated with M1.4, X1.2 and M5.9 flares at AR10691 at 6:54, $12: 30$ and 16:54, respectively.

The annual intensity plot shows an expected shape which reflects the solar activity level. The annual $\mathrm{Fe} / \mathrm{C}$ and $\mathrm{Fe} / \mathrm{O}$ ratios show fairly stable, albeit slightly declining trend with the quiet-time years 1996 and 2007-2009 excluded. During the quiet years the $\mathrm{Fe} / \mathrm{C}$ and $\mathrm{Fe} / \mathrm{O}$ ratios have average values of about 0.04 and 0.004 , respectively, which are much lower than for example the value for 

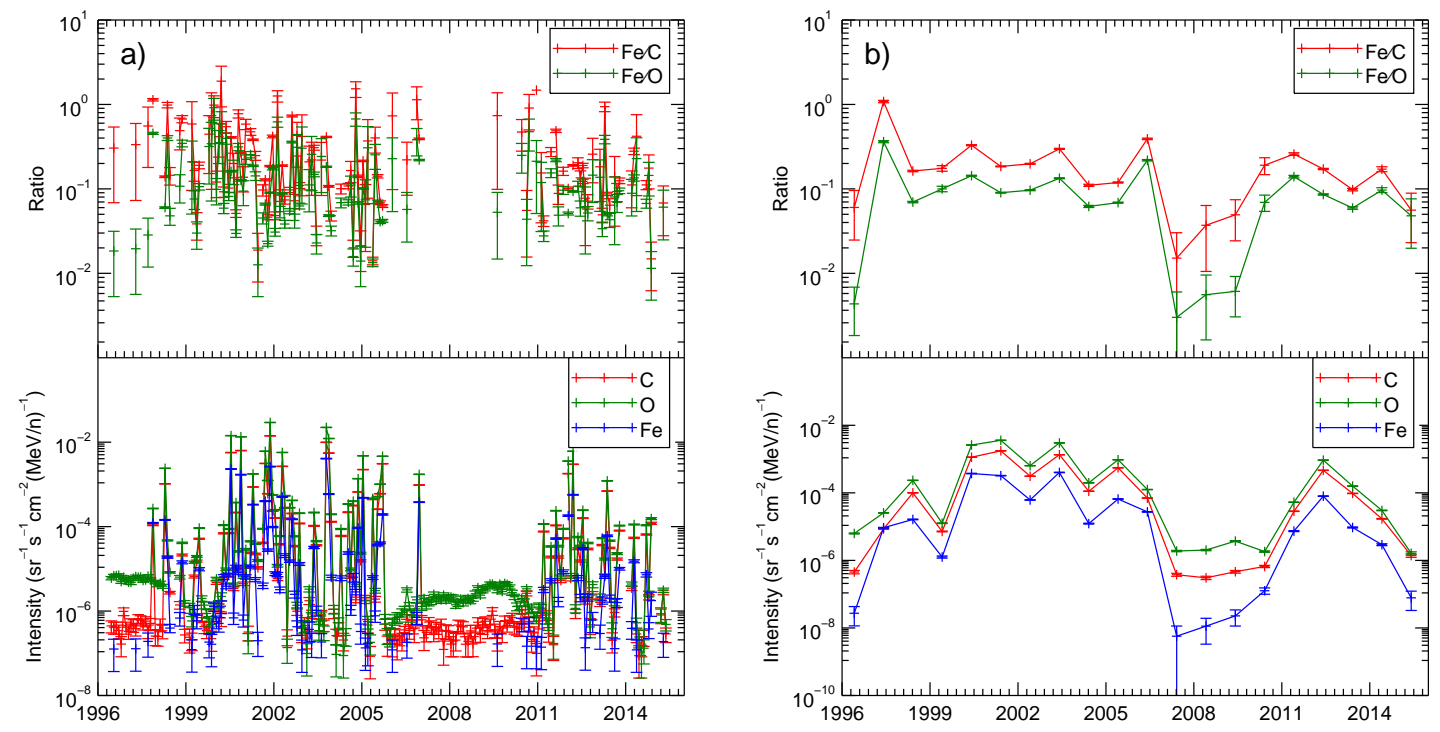

Figure 1: a) Monthly intensities of 5-15 MeV/n C, O and $\mathrm{Fe}$ and the ratios of $\mathrm{Fe}$ to $\mathrm{C}$ and $\mathrm{Fe}$ to $\mathrm{O}$; b) Annual intensities of 5-15 MeV/n C, $\mathrm{O}$ and $\mathrm{Fe}$ and the ratios of $\mathrm{Fe}$ to $\mathrm{C}$ and $\mathrm{Fe}$ to $\mathrm{O}$.

$\mathrm{Fe} / \mathrm{O}$ in corona $\left(0.186\right.$, measured at $\left.1.4 \cdot 10^{6} \mathrm{~K}[\mathrm{Q}]\right)$ or the values for $\mathrm{Fe} / \mathrm{O}$ in slow and fast solar wind ( 0.120 and 0.092 , measured at $1 \mathrm{keV}$ and $2 \mathrm{keV}$, respectively) [ए0]].

Figures $\square$ a) and b) show the daily average intensities of $5-15 \mathrm{MeV} / \mathrm{n}$ Fe versus the average daily intensities of 5-15 MeV/n C and O. Days of solar cycles 23 and 24 are shown in red and blue, respectively. Days with one or less counts of either $\mathrm{C}, \mathrm{O}$ or Fe have been omitted from the figures. The overall number of points in both figures is $499 ; 381$ of them belong to solar cycle 23 and only 118 to solar cycle 24 , giving a ratio of $381 / 118 \approx 3.2$. The diffence in solar cycle lengths $(11.7$ years of cycle 23 versus 7.4 observed years of cycle 24 , giving a ratio of 1.6) is not nearly enough for an explanation for the deficiency of days with sufficient heavy ion counts during the current solar cycle.

Figure [ ] a) shows the distribution of daily average intensities of $\mathrm{C}, \mathrm{O}$ and $\mathrm{Fe}$, for solar cycles 23 (in red) and 24 (in blue). All distributions have been corrected for the amount of time the SOHO/ERNE has been offline during the measurement period. In addition, the distributions of solar cycle 23 have been divided by the ratio $t_{S C 23} / t_{S C 24}$, where $t_{S C 23}$ is the length of solar cycle 23 and $t_{S C 24}$ is the length of solar cycle 24 up to May 31, 2015. Even after these corrections, the intensity distributions have different heights, as was expected concerning the large difference in the number of days with sufficient statistics. Nonetheless, the shapes of the distributions are quite similar for cycles 23 and 24.

The ratio distributions shown in Figure [ $\mathrm{b}$ ) have been corrected and scaled in the same way as the ones in Figure [ ] a). Besides the different heights, the distributions also seem to have somewhat different shapes for solar cycles 23 and 24 . The distributions of cycle 23 have narrow maximae, whereas the distributions of cycle 24 could be interpreted as two-peak structures, with minimae between the peaks at values of about 0.3 and 0.2 for $\mathrm{Fe} / \mathrm{C}$ and $\mathrm{Fe} / \mathrm{O}$, respectively. These values 

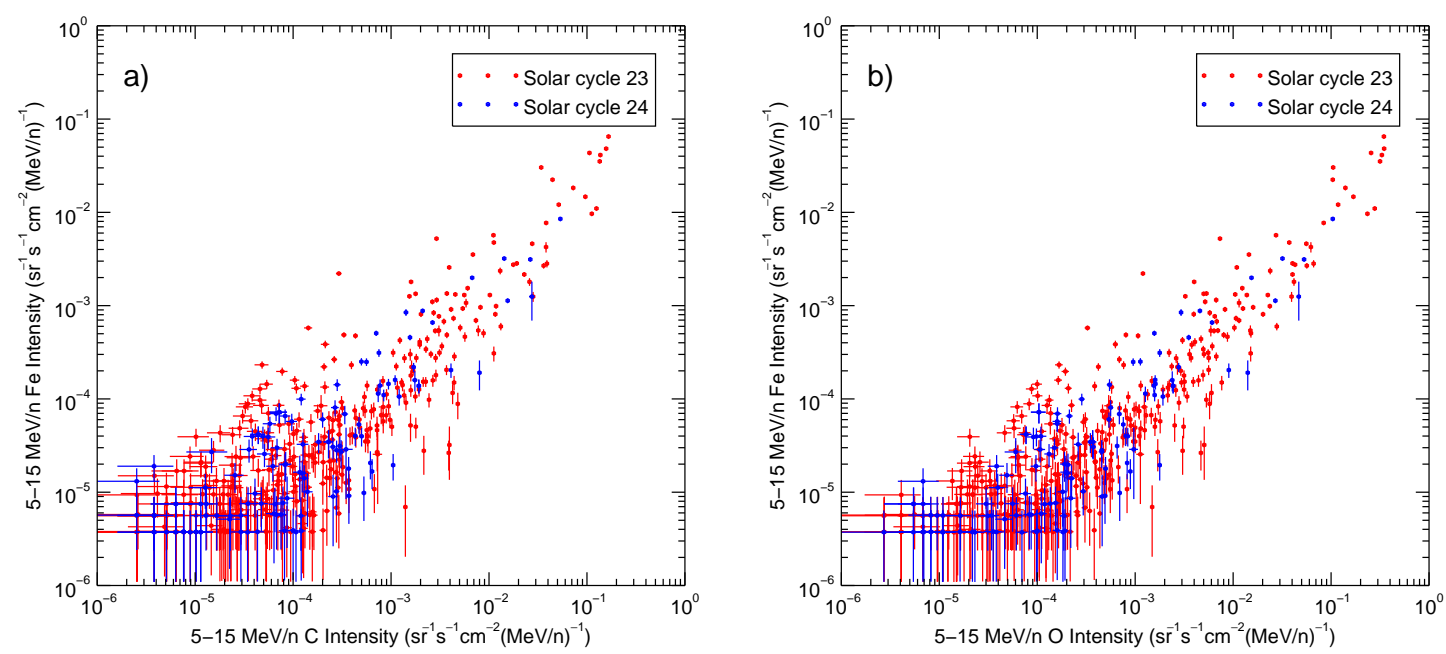

Figure 2: a) Average daily 5-15 MeV/n Fe intensities versus average daily C intensities; b) Average daily 5-15 MeV/n Fe intensities versus average daily $\mathrm{O}$ intensities. In both figures, solar cycle 23 is shown in red and solar cycle 24 in blue. Only points with more than one count of $\mathrm{C}, \mathrm{O}$ and $\mathrm{Fe}$ each have been included.
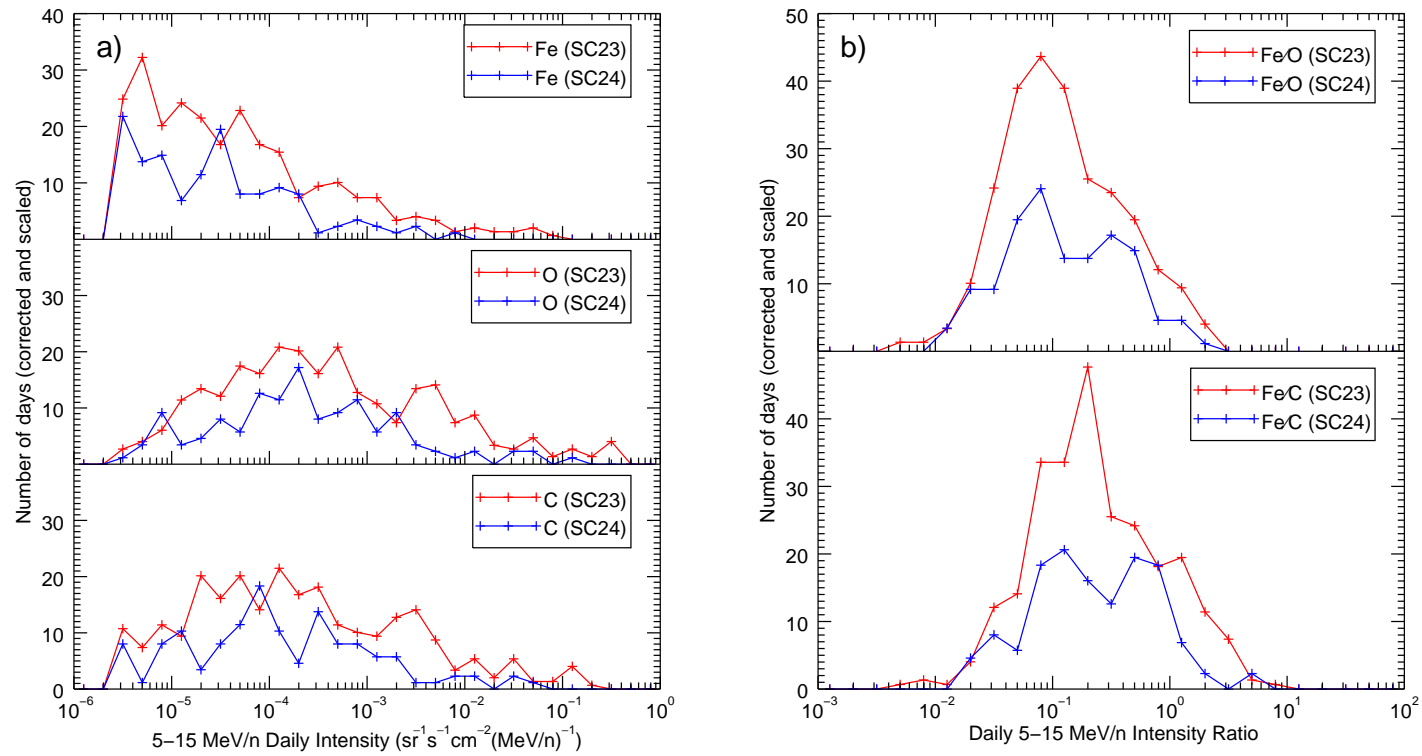

Figure 3: a) Distribution of daily average intensities of $\mathrm{C}, \mathrm{O}$ and $\mathrm{Fe}$. b) Distribution of the daily ratios of $\mathrm{Fe}$ to $\mathrm{C}$ and $\mathrm{Fe}$ to $\mathrm{O}$. For both a) and b), only days with more than one count of $\mathrm{C}, \mathrm{O}$ and $\mathrm{Fe}$ have been included in the plot. Solar cycle 23 is shown in red and solar cycle 24 in blue. All distributions have been corrected for the time SOHO/ERNE has been offline, and the SC23 distributions have been scaled down to account for the difference in durations of SC23 and SC24 (until 31.5.2015). 

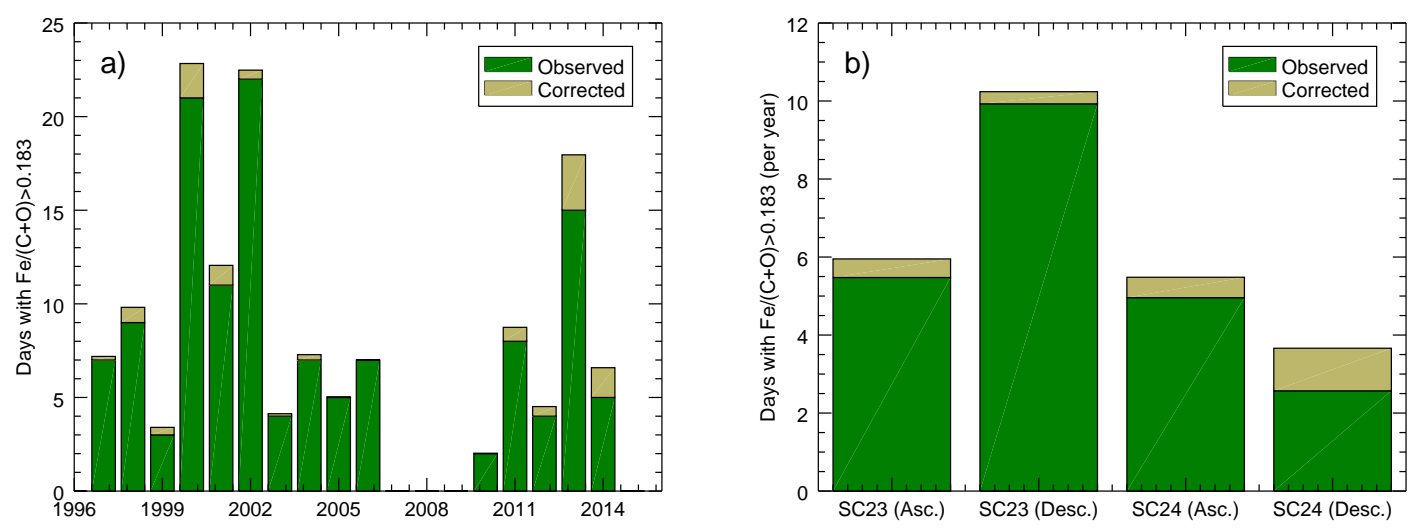

Figure 4: a) Annual number of days with $\mathrm{Fe} /(\mathrm{C}+\mathrm{O})>0.183$. b) Number of days with $\mathrm{Fe} /(\mathrm{C}+\mathrm{O})>0.183$ during the rising and falling phases of solar cycles 23 and 24 in units of $a^{-1}$. For both a) and b), the corrections account for the time SOHO/ERNE has been offline during each year or phase.

are close to the relative abundances in gradual SEP events measured at 5-12 MeV/n, $0.288(\mathrm{Fe} / \mathrm{C})$ and $0.134(\mathrm{Fe} / \mathrm{O})[\mathbb{W}]$. Using different binning for the distributions changes the shapes somewhat, but the minimae do not disappear completely. In the context of the two classes of SEP events, this two-peak shape is expected, and similar results have been reported in several similar surveys (e.g. [回])

\section{Event selection}

As the purpose is to study events with enhanced abundances of heavy elements, we used the following two criteria to select events: 1) Daily counts of $\mathrm{C}, \mathrm{O}$ and $\mathrm{Fe}$ must each be greater than one; 2) The ratio of $\mathrm{Fe} /(\mathrm{C}+\mathrm{O})$ must be higher than 0.183 , which is the corresponding value of gradual solar particle events in the $5-12 \mathrm{MeV} / \mathrm{n}$ region as reported by [ए]]. There were 130 days that fulfilled the criteria, 96 of them during solar cycle 23, and 34 during cycle 24. Figure 7 a) shows the number of selected days for each year, with corrections for the time SOHO/ERNE has been offline during each year. Figure $\boxplus b$ ) shows the number of selected days in units of year ${ }^{-1}$ for the two cycles, divided into periods before and after the cycle maximum, with similar corrections as Figure $\$$ a). From this figure it is clear that the two solar cycles are different concerning the heavy elements; the rate of occurrence of Fe-enhancements during the phase after the cycle maximum in the current cycle is less than half of what it was during the previous cycle. However, if we divide the rate of occurrence of Fe-enhancements with the number of days observed with more than one counts of $\mathrm{C}, \mathrm{O}$ and $\mathrm{Fe}$, the situation changes: the percentages of Fe-enriched days during the cycle phases are $0.44,0.23,0.30$ and 0.23 for cycles 23 and 24 , respectively. In this context the phases after cycle maximums have been very similar, whereas the phase before the maximum of cycle 24 had a somewhat decreased number of Fe-enriched days.

To study complete SEP events instead of regarding each day as a separate event, we made a visual scan of the SOHO/ERNE proton data and used previous catalogues of SEP events ([12], [13]) to associate the Fe-enriched days with SEP events. In addition, we used the SOHO/LASCO CME 
catalogue $^{1}$, GOES flare data ${ }^{2}$ and radio data from Wind/WAVES ${ }^{3}$ to get CME, flare and type III burst associations for each event. In the end, we managed to identify 85 events with one or more days with Fe enrichment; 63 of them occurred during solar cycle 23 and 22 during cycle 24.

\section{Properties of heavy ions}

Figure $\mathrm{B}$ shows the event-averaged abundance ratios of $\mathrm{N}, \mathrm{Ne}, \mathrm{Mg}, \mathrm{Si}, \mathrm{S}$ and $\mathrm{Fe}$ to $\mathrm{O}$ versus the ratio of $\mathrm{Fe}$ to $\mathrm{C}$. Events occurring during solar cycles 23 and 24 are shown in red and blue, respectively. Linear fits in log-log scales to both sets of events and the slopes of the fits are also shown. Only events with 4 or more counts are included. The slopes of events of cycle 24 get steeper when moving from N/O towards $\mathrm{Fe} / \mathrm{O}$, as reported before (e.g., [44, [5]]); $\mathrm{Ne} / \mathrm{O}$ is an exception to this trend, but it seems to have large errors as well. Events of cycle 23 do not exhibit this behavior as clearly. Another noteworthy thing in Figure $\square$ is that all but one of the events of cycle 24 have $\mathrm{Fe} / \mathrm{C}$ values of less than one, whereas there are many events that occurred during solar cycle 23 that had $\mathrm{Fe} / \mathrm{C}$ values between 1 and 10 .
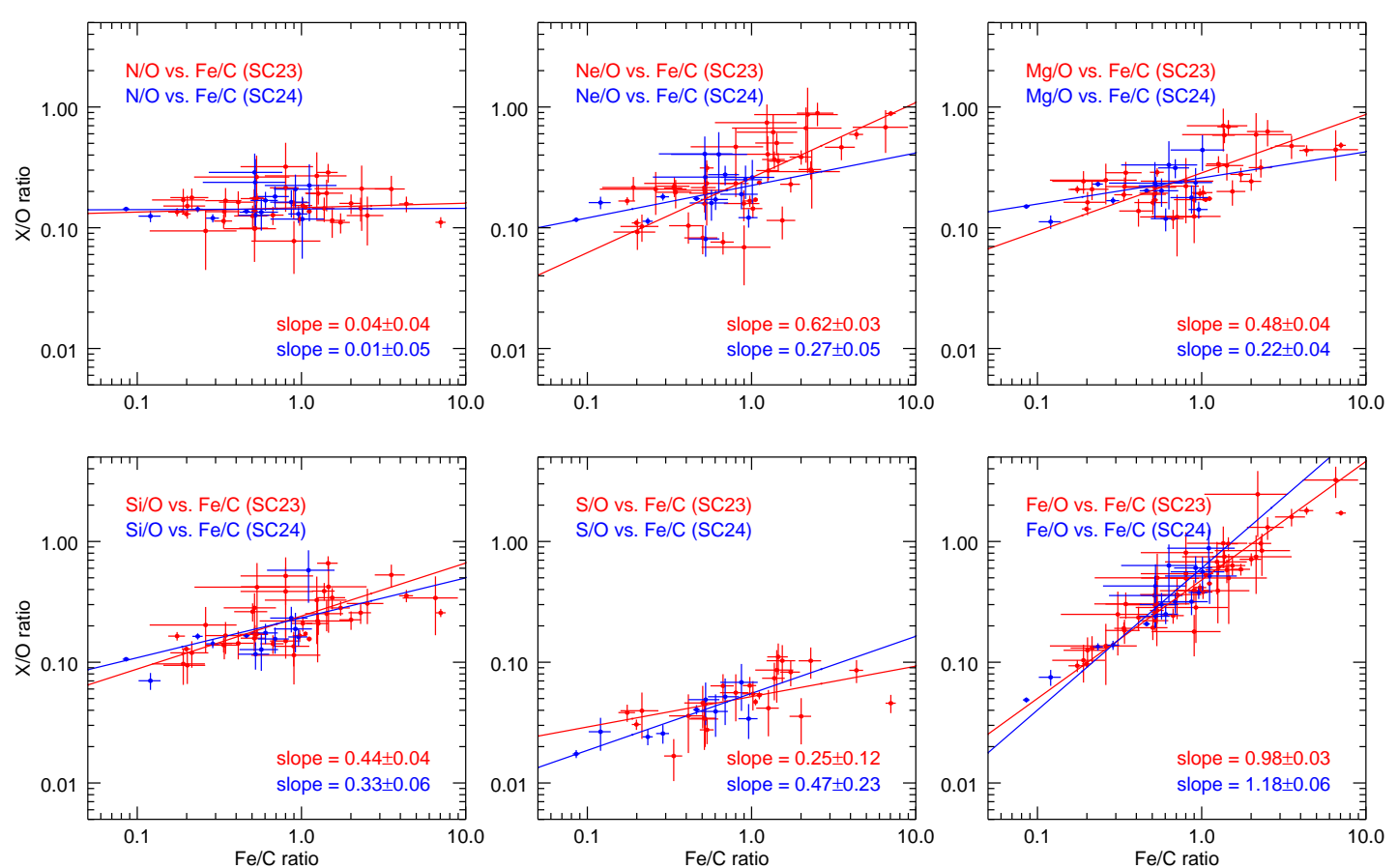

Figure 5: Ratios of $\mathrm{N}, \mathrm{Ne}, \mathrm{Mg}, \mathrm{Si}, \mathrm{S}$ and $\mathrm{Fe}$ to $\mathrm{O}$ versus the ratio of $\mathrm{Fe}$ to $\mathrm{C}$ in the selected SEP events. Events occurring during solar cycles 23 and 24 are shown in red and blue, respectively. The lines are linear fits to both sets of events.

\footnotetext{
${ }^{1}$ Available online at http://cdaw.gsfc.nasa.gov/CME_list/index.html

${ }^{2}$ Available online at ftp://ftp.ngdc.noaa.gov/STP/space-weather/solar-data/solar-features/ solar-flares/x-rays/goes/

${ }^{3}$ Available online at ftp://stereowaves.gsfc.nasa.gov/
} 


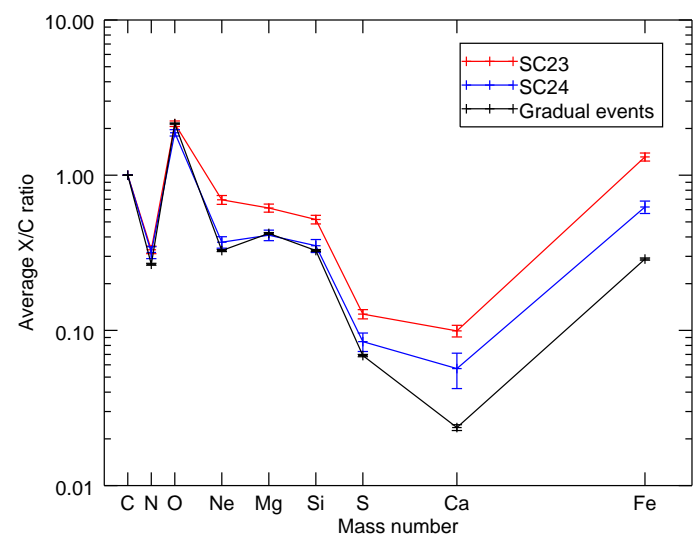

Figure 6: Average abundances of heavy ions in the iron-rich events (relative to $\mathrm{C}$ ) as a function of mass number. Average values for the events occurring during solar cycles 23 and 24 are shown in red and blue, respectively. The values for gradual SEP events reported in [U]] are shown as a comparison (in black).

Figure 6 shows the unweighted average abundances (relative to C) of the events in this study as a function of mass. Again, only events with 4 or more counts have been included in the calculation of averages. Values for gradual SEP events reported in [W] are shown as a comparison. There are some interesting differences in the abundances between the two solar cycles. The values for $\mathrm{C}, \mathrm{N}$ and $\mathrm{O}$ are similar for both solar cycles and for the gradual events, but from $\mathrm{Ne}$ up to $\mathrm{Fe}$ the values for cycle 23 are all enriched by a factors between 1.5 and 2 compared to cycle 24 . The values of cycle 24 are similar to the values of gradual events from $\mathrm{C}$ up to $\mathrm{S}$, but $\mathrm{Ca}$ and Fe are about two times higher for the cycle 24 .

\section{Summary}

We surveyed the SOHO/ERNE heavy ion data from the beginning of solar cycle 23 until the end of May 2015, i.e. after the maximum of solar cycle 24. The long observation period allowed us to study and compare the properties of heavy ions in SEP events during the two solar cycles. We found that the overall rate of heavy ion enrichments has been slower during cycle 24 than during cycle 23 , but that it can be explained by the smaller total solar activity during the cycle 24 . We searched for enhancements in the daily $\mathrm{Fe} /(\mathrm{C}+\mathrm{O})$ ratio and made a selection of SEP events that had days with $\mathrm{Fe} / \mathrm{C}+\mathrm{O}$ ) $>0.183$ (twice the value for gradual SEP events) during them. We found that those of the events that occurred during solar cycle 23 had larger average enhancements of heavy ions than those that occurred during solar cycle 24.

\section{Acknowledgments}

We gratefully acknowledge the use of data made available at the GSFC and NOAA STP web services. O. R. wishes to thank the Vilho, Yrjö and Kalle Väisälä foundation for financial support. 


\section{References}

[1] S. E. Forbush, Three unusual cosmic-ray increases possibly due to charged particles from the sun, Phys. Rev., 70, 771, 1946

[2] J. P. Wild, S. F. Smerd \& A. A. Weiss, Solar bursts, Annu. Rev. Astron. Astrophys., 1, 291, 1963

[3] D. V. Reames, The two sources of solar energetic particles, Space Sci. Rev., 175, 53, 2013

[4] D. V. Reames, T. T. von Rosenvinge \& R. P. Lin, Solar ${ }^{3}$ He-rich events and nonrelativistic electron events: a new association, Astrophys. J., 292, 716, 1985

[5] D. V. Reames, Bimodal abundances in the energetic particles of solar and interplanetary origin, Astrophys. J., 330, L71, 1988

[6] G. M. Mason, J. E. Mazur \& J. R. Dwyer, ${ }^{3}$ He enhancements in large solar energetic particle events, Astrophys. J., 525, L133, 1999

[7] J. Torsti et al., Solar particle event with exceptionally high ${ }^{3}$ He enhancement in the energy range up to $50 \mathrm{MeV}$ nucleon $^{-1}$, Astrophys. J., 573, L59, 2002

[8] A. J. Tylka et al., Shock geometry, seed populations, and the origin of variable elemental composition at high energies in large gradual solar particle events, Astrophys. J., 625, 474, 2005

[9] U. Feldman \& K. G. Widing, Elemental abundances in the solar upper atmosphere derived by spectroscopic means, Space Sci. Rev., 107, 665, 2003

[10] R. von Steiger et al., Composition of quasi-stationary solar wind flows from Ulysses/Solar Wind Ion Composition Spectrometer, J. Geophys. Res., 105, A12, 27217, 2000

[11] D. V. Reames, Coronal abundances determined from energetic particles, Adv. Space Res., 15(7), 41, 1995

[12] H. V. Cane, I. G. Richardson \& T. T. von Rosenvinge, A study of solar energetic particle events of 1996-2006: Their composition and associations, J. Geophys. Res., 115, A08101, 2010

[13] R. Vainio et al., The first SEPServer event catalogue $\sim 68-M e V$ solar proton events observed at 1 AU in 1996-2010, J. Space Weather Space Clim., 3, A12, 2013

[14] D. V. Reames, J. P. Meyer \& T. T. von Rosenvinge, Energetic particle abundances in impulsive solar flare events, Astrophys. J. Supp., 90, 649, 1994

[15] G. M. Mason et al., Abundances of heavy and ultraheavy ions in ${ }^{3}$ He-rich solar flares, Astrophys. J., 606, 555, 2004 